\title{
The selective blockade of metabotropic glutamate receptor-5 attenuates fat accumulation in an in vitro model of benign steatosis
}

\author{
Andrea Ferrigno, Clarissa Berardo, Laura Giuseppina Di Pasqua, Marta Cagna, Veronica Siciliano, \\ Plinio Richelmi, Mariapia Vairetti
}

Department of Internal Medicine and Therapeutics, Unit of Cellular and Molecular Pharmacology and Toxicology, University of Pavia, Italy

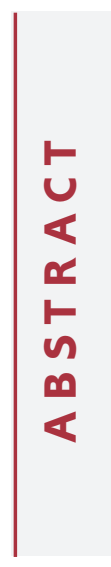

\begin{abstract}
It has been previously found that the blockade of metabotropic glutamate receptor type 5 (mGluR5) protects against hepatic ischemia/reperfusion injury and acetaminophen toxicity. The role of mGluR5 in NAFLD has not yet been elucidated. Here, we evaluated the effects of mGluR5 blockade in an in vitro model of steatosis. HepG2 cells were pre-incubated for $12 \mathrm{~h}$ with an mGluR5 agonist, a negative allosteric modulator (DHPG and MPEP, respectively) or vehicle, then treated with $1.5 \mathrm{mM}$ oleate/palmitate $(\mathrm{O} / \mathrm{P})$ for another $12 \mathrm{~h}$. Cell viability was evaluated with the MTT assay; fat accumulation was measured using the fluorescent dye Nile red; SREBP1 , PPAR- $\alpha$, iNOS and Caspase-3 protein expression were evaluated by Western blot; NFkB activity was evaluated as pNFkB/NFkB ratio. mGluR5 modulation did not alter cell viability in O/P-incubated cells; MPEP prevented intracellular lipid accumulation in O/P treated cells; MPEP administration was also associated with a reversion of O/P-induced changes in SREBP-1 and PPAR- $\alpha$ expression, involved in free fatty acid metabolism and uptake. No changes were observed in iNOS and caspase-3 expression, or in NFkB activity. In conclusion, mGluR5 pharmacological blockade reduced fat accumulation in HepG2 cells incubated with O/P, probably by modulating the expression of SREBP-1 and PPAR- $\alpha$.
\end{abstract}

Key words: NAFLD; mGluR5; MPEP, oleate/palmitate; HepG2; SREBP-1; PPAR- $\alpha$.

Correspondence: Clarissa Berardo, Department of Internal Medicine and Therapeutics, Unit of Cellular and Molecular Pharmacology and Toxicology, University of Pavia, 27100 Pavia, Italy. Tel. +39.0382 .986873$.

E-mail: clarissa.berardo01@universitadipavia.it

Contributions: $\mathrm{AF}$ and $\mathrm{CB}$ contributed equally. All authors have contributed significantly: AF, formal analysis and original manuscript preparation; AF, MV, conceptualization; CB, LGDP, VS, MC, methodology, software, validation and data curation; MV, PL, CB, LGDP, manuscript revision. All authors have read and agreed to the published version of the manuscript.

Funding: The present research and publication costs were funded by Ministry of Health, "Young Researchers" 2011-2012 (project number: GR-2011-02349273).

Conflict of interest: All authors declare no conflict of interest related to this publication.

Availability of data and materials: The data used to support the findings of this study are available from the corresponding author upon request.

Ethics approval: No ethical committee approval was required for this study, as it does not involve human participants or animals. 


\section{Introduction}

Nonalcoholic fatty liver disease (NAFLD) is a liver disorder caused by intrahepatic fat overload, usually associated with obesity, insulin resistance, hypertension and dyslipidemia. NAFLD definition includes a vast array of hepatic conditions, such as uncomplicated fat accumulation or fatty liver, steatohepatitis (NASH), fibrosis and cirrhosis. In Western countries, NAFLD prevalence is about $20-40 \%$ in general population, rising up to $75 \%$ in obese or diabetic people, even though NASH is present in a limited portion of subjects. ${ }^{1}$ The pathogenesis of NAFLD has been studied in in vitro models, animal models and in patients. According to the "two hit hypothesis", the first hit consists in the hepatic free fatty acid (FFA) accumulation that, in turn, triggers inflammatory cascades (the second hit) resulting in a chronic inflammatory state and finally leading to NASH. ${ }^{2,3}$ Nowadays, the multiple-hit hypothesis is prevalent; in fact, a wide range of parallel processes are deemed to be involved in the first manifestation and in the progression of NAFLD, including insulin resistance, mitochondrial dysfunction, endoplasmic reticulum stress, activation of inflammatory pathways, adipose tissue dysfunction, genetic variants, epigenetic factors, nutritional habits and altered gut microbiome. However, at the liver level, FFA overflow is considered the triggering factor. ${ }^{4}$ The presence of metabotropic glutamate receptors (mGluRs) in peripheral tissues, including those not originating from neural crests, has been demonstrated in numerous studies. ${ }^{5}$ mGluRs have been found in organs and tissues, such as: pancreas, liver, stomach mucosa, human testes and ovaries. ${ }^{6-10}$ The presence of mGluRs in the liver has been demonstrated for the first time in 1997, when the mGluR competitive agonists 1-Amino-1,3-dicarboxycyclopentane (ACPD) and quisqualate were shown to induce inositol monophosphate formation in primary rat hepatocytes. ${ }^{11}$ Successively, Storto and colleagues confirmed that mGluR5 is expressed in rat primary hepatocytes and hepatoma HepG2 cells using Western blot, immunohistochemistry and RT-PCR analysis; the homologue mGluR1 was not found in the same cells. ${ }^{7}$ The role of mGluRs in liver injury was also investigated: mGluR5 activation promoted ischemic damage in primary hepatocytes, while its pharmacological blockade using the selective mGluR5 negative allosteric modulator 2-methyl-6-(phenylethynyl)pyridine (MPEP), as well as mGluR5 gene depletion, provided primary hepatocytes a higher resistance to hypoxic injury. ${ }^{7,12}$ The blockade of mGluR5 protected also against oxidative injury. MPEP administration was associated with a reduced ROS production, lipid peroxidation and protein $\mathrm{SH}$ groups oxidation in isolated rat hepatocytes treated with $0.5 \mathrm{mM}$ tert-butyl hydroperoxide. The improvement of oxidative stress was not matched by an increase in GSH stores, suggesting that the mGluR5 negative modulator did not act by scavenging the toxic acetaminophen metabolite but by modulating inflammatory pathways. In fact, the administration of MPEP to acetaminophen-intoxicated mice reduced iNOS activity in liver homogenates, suggesting that mGluR5 may be involved in the activation of inflammatory processes. ${ }^{13}$ More recently, it has been shown in ex vivo models of hepatic cold and warm ischemia that mGluR5 blockade stops mGluR5 hyperactivation and reduces the expression of inflammatory factors such as TNF- $\alpha$ and iNOS. ${ }^{14}$ The mechanism underlying the increase in glutamate release involved in mGluR5 hyperactivation has not yet been completely elucidated; however, an increase in glutamate release has been observed under hypoxic conditions in isolated rat hepatocytes ${ }^{7}$ and in a rat portal vein ligation model. ${ }^{15}$ The resulting glutamate accumulation was considered the culprit for mGluR5 hyperactivation involved in the promotion of liver ischemic injury. ${ }^{14,16}$ Recently, it has been shown that an increased glutamate secretion also occurs in an alcoholinduced steatosis model, resulting in mGluR5s hyperactivation and abnormal fat accumulation in hepatocytes. ${ }^{17}$ No study is currently available on the mGluR5 role in the development of NAFLD; then, the aim of this work was to elucidate the role of mGluR5 in an in vitro model of steatosis in HepG2 cells. Since we have previously shown that mGluR5 blockade is associated with the downregulation of inflammatory proteins, we opted for an in vitro model in which fat accumulation was associated with low inflammatory levels, to focalize our attention on the role of mGluR5 in FFA metabolism modulation.

\section{Materials and Methods}

\section{Substances}

Sodium palmitate (\#P9767) and sodium oleate (\#O5701), fatty acid free bovine serum albumin (BSA, \#A7030), cell lysis reagent (\#C3228), protease inhibitor cocktail (\#P8340), Nile red (\#19123), minimum essential medium EAGLE (\#M4655), sodium pyruvate (\#S8636), penicillin-streptomycin-amphotericin antibiotic mixture (\#A5955) 3-(4,5-dimethylthiazol-2-yl)-2,5-diphenyltetrazolium bromide (MTT, \#M2128) and (S)-3,5-dihydroxphenylglycine (DHPG, \#D3689) were purchased from Sigma-Aldrich (Milan, Italy). 2',7'-dichlorofluorescein diacetate ( $\mathrm{H}_{2} \mathrm{DCF}-\mathrm{DA}$, \#D-399) and Hoechst 33342 (\#H1399) were provided respectively from Molecular Probes and Life Technologies (Invitrogen, Thermo Fisher Scientific, Milan, Italy). 2-methyl-6-(phenylethynyl)pyridine hydrochloride (MPEP, \#1212) was acquired by Tocris Bioscence (Bristol, UK). L-glutamine was from Biological Industries (Cromwell, CT, USA), while fetal bovine serum (FBS) from South America was purchased from Corning Life Science (\#35-079-CV, Oneonta, NY, USA). The mouse monoclonal antitubulin (\#T9096) was from Sigma-Aldrich, the rabbit polyclonal anti-mGluR5 (\#AB5675) was from EDM Millipore (Merk Life Science, Milan, Italy). Santa Cruz Biotechnology (Dallas, TX, USA) provided us the rabbit polyclonal anti-actin (\#sc-1616) and the following mouse monoclonal antibodies: anti-sterol regulatory element-binding protein 1 (SREBP-1, \#sc-365513), anti-peroxisome proliferator-activated receptor alpha (PPAR-a, \#sc-398394), anti-NFkB p65 (\#sc-514451), anti-p-NFkB p65 (\#sc-136548). The mouse monoclonal anti-cleaved caspase- 3 was from Cell Signaling Technology (Leiden, The Netherlands). The rabbit polyclonal antiinducible nitric oxide synthase (iNOS, \#160862) was from Cayman Chemical (Ann Arbor, MI, USA).

\section{Cell culture}

HepG2 cells were grown in minimum essential medium EAGLE supplemented with 10\% FBS, 1\% L-glutamine, $1 \%$ sodium pyruvate and $1 \%$ antibiotic, at $37^{\circ} \mathrm{C}$ and $5 \% \mathrm{CO}_{2}$. HepG2 cells were seeded in 96-well plate at 50.000 cells/well and were used at $75 \%$ of confluence. For Western blot analyses, $2 \times 10^{6}$ cells were seeded onto $10 \mathrm{~cm}$ Petri dishes.

\section{Treatments}

On the day of the experiment, cells were washed once with sterile phosphate-buffered saline (PBS; sodium phosphate dibasic anhydrous $40 \mathrm{mM}$, sodium dihydrogen phosphate monohydrate 10 $\mathrm{mM}, 700 \mathrm{mM}$ sodium chloride, $\mathrm{pH}$ 7.4) and pre-treated for $12 \mathrm{~h}$ with the mGluR5 orthosteric agonist DHPG $100 \mu \mathrm{M}$ alone or in combination with the negative allosteric modulator MPEP at 0.3 $\mu \mathrm{M}$. Palmitic and oleic acids, provided as sodium salts, were dissolved in PBS (stock solution $50 \mathrm{mM}$ ). After the pharmacological pre-treatment, a mixture of oleate:palmitate $(\mathrm{O} / \mathrm{P}$, molar ratio $2: 1)$ at final concentration of $1.5 \mathrm{mM}$ was diluted in a serum-free medium supplemented with $1 \%$ fatty acid free BSA, with or without the 
previous pharmacological treatments, and used to incubate HepG2 cells for $12 \mathrm{~h}$. Control cells were incubated in serum-free medium supplemented with $1 \%$ fatty acid-free BSA. Three independent experiments were realized; each condition was reproduced in duplicate.

\section{Cell viability}

Viability of HepG2 cells treated with fatty acids and mGluR5 modulators was assessed by the colorimetric MTT assay, as previously described. ${ }^{18}$ Briefly, cells were washed once with PBS and MTT $1.2 \mathrm{mM}$ diluted in serum and BSA free fresh medium was added to each well. For negative control, $4 \mu 1$ of $25 \%$ Tryton X per well were added prior to the treatment with MTT. After $2 \mathrm{~h}$ of incubation at $37^{\circ} \mathrm{C}$ at $5 \% \mathrm{CO}_{2}$, formazan crystals were dissolved with DMSO. The absorbance was measured at $540 \mathrm{~nm}$ using a microplate reader (Bio-Rad, Hercules, CA, USA).

\section{Lipid accumulation}

The lipid content in HepG2 cells was determined employing the vital lipophilic dye Nile red, calculated in function of labeled dsDNA by means of Hoechst 33342. At the end of treatment, cells were washed once with PBS and incubated with $1 \mu \mathrm{M}$ Nile red and Hoechst, for $15 \mathrm{~min}$. Then, cells were washed once with PBS and images were taken using ZOE (Bio-Rad). Images were then analyzed by ImageJ software, using the subtraction background method $(50 \%)$.

\section{Reactive oxygen species production}

Oxidative stress in HepG2 cells was obtained using the conversion of the 2', 7'-dichlorofluorescein diacetate $\left(\mathrm{H}_{2} \mathrm{DCF}-\mathrm{DA}\right)$ to a fluorescent molecule, the 2',7'-dichlorofluorescein (DCF), compared to Hoechst 33342 stained nuclei. Cells were incubated with a PBS solution containing $\mathrm{H}_{2}$ DCF-DA and Hoechst, both at $1 \mu \mathrm{M}$, for $15 \mathrm{~min}$ at $37^{\circ} \mathrm{C}$ and $5 \% \mathrm{CO}_{2}$. Then, cells were washed once with PBS and observed at the fluorescent cell imager ZOE (BIORAD). Images were analyzed by ImageJ software, using the subtraction background method (100\%).

\section{Western blot}

HepG2 cells were treated with $500 \mu \mathrm{L}$ of CelLytic buffer supplemented with protease inhibitor cocktail $(10 \mu \mathrm{L} / \mathrm{mL})$ and centrifuged at $15000 \mathrm{~g}$ for $10 \mathrm{~min}$. The protein concentration was determined by Lowry assay. Cell extracts containing the same amount of proteins were separated in SDS-PAGE on 7, 10 or $12 \%$ acrylamide gels, and transferred to PVDF membrane, as previously described. ${ }^{14}$ Unspecific sites were blocked for $2 \mathrm{~h}$ with 5\% BSA in tris-buffered saline (TBS, $20 \mathrm{mM}$ Tris/ $\mathrm{HCl}, 500 \mathrm{mM}$ sodium chloride, $\mathrm{pH} 7.5,0.1 \%$ Tween 20 ) at $4^{\circ} \mathrm{C}$. The membranes were incubated with primary antibodies overnight at $4{ }^{\circ} \mathrm{C}$, under gentle agitation, while secondary antibodies were incubated at the same condition for $1 \mathrm{~h}$. Primary antibodies against mGluR5, SREBP-1, PPAR- $\alpha$, iNOS, NFkB p65 and phospho-NFkB p65, cleaved caspase-3, tubulin and actin were used at 1:1000 dilution. Mouse peroxidase-conjugated secondary antibody was used at 1:1000 dilution for SREBP-1, PPAR- $\alpha$ and NFkB, as well rat peroxidase-conjugated secondary antibody for cleaved caspase-3. Immunostaining was revealed with BIO-RAD Chemidoc XRS+. Bands intensity quantification was performed by Bio-Rad Image Lab software 5.

\section{Statistical analysis}

Statistical analysis was performed using R Statistical software (v. 3.3.0) and the graphical interface R Studio (v. 1.0.143). The normality and homogeneity of variances were verified employing Shapiro's test and Levene's test, respectively. In the majority of cases, data had normal distribution curves and ANOVA and Tukey's HSD Test used for multiple comparisons. The KruskalWallis non-parametric test was used for data with non-homogeneous variances; in this case, Dunn's test was used for multiple comparisons.

\section{Results}

\section{mGluR5 protein expression in HepG2}

mGluR5s have been previously found in rat and mouse hepatocytes $^{11,19}$ and in HepG2. ${ }^{7}$ However, considering that the mGluR5 expression on human hepatoma cells has not been extensively investigated, we decided to evaluate its expression in the hepatoma cell lines available in our laboratory (HepG2 and Huh7.5) in comparison with the receptor expression in rat cortex. As expected, mGluR5 is much more expressed in rat cortex than in hepatoma cells, even though it was significantly expressed in HepG2 cells (Figure 1). The mGluR5 expression in Huh7.5 cells was considered too scarce.

\section{mGluR5 blockade reduces fat accumulation in HepG2 treated with $1.5 \mathrm{mM} \mathrm{O} / \mathrm{P}$ mix}

Based on mGluR5 expression levels, HepG2 were chosen for these experiments. HepG2 were pre-incubated for $12 \mathrm{~h}$ with 100 $\mu \mathrm{M}$ of mGluR5 agonist DHPG alone (DHPG group) or with MPEP

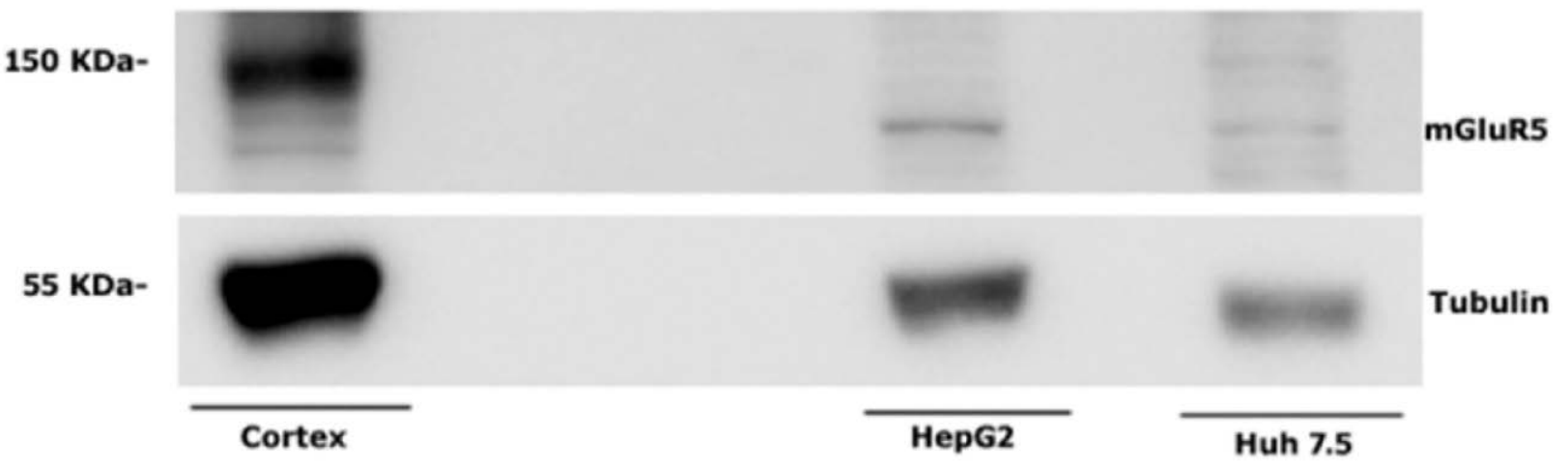

Figure 1. Expression of mGluR5 in rat cortex, HepG2 and Huh7.5 hepatoma cells. 
$0.3 \mu \mathrm{M}$ (MPEP group); then, $\mathrm{O} / \mathrm{P}$ mixture was added to both DHPG and MPEP groups for $12 \mathrm{~h}$. In MIX group, HepG2 were incubated with $\mathrm{O} / \mathrm{P}$ mixture and received no drug treatment; CTRL group consisted in untreated control HepG2. A significant reduction in the MIX group cell viability, as evaluated with the MTT assay, was found in comparison with the CTRL group; no other significant difference was observed (Figure 2A). Fat accumulation was evaluated as Nile red/Hoechst ratio using Image J. The administration of $1.5 \mathrm{mM} \mathrm{O} / \mathrm{P}$ mixture to HepG2 induced a significant increase in fat content in the MIX group compared to CTRL cells. The activation of mGluR5 in $\mathrm{O} / \mathrm{P}$-treated cells did not result in a further increase in fat accumulation when comparing the DHPG group with untreated MIX cells, suggesting that either mGluR5 or the downstream mechanism involved in fat accumulation was fully activated by the O/P mixture alone. Finally, the mGluR5 blockade in MPEP group reduced significantly FFA accumulation when comparing MPEP cells to both MIX and DHPG groups (Figure 2 B-F). The expression of mGluR5 was also evaluated but no significant difference was found between the experimental groups (Supplementary Figure 1).

\section{mGluR5 blockade is associated with a reversion of $\mathrm{O} / \mathrm{P}$ mixture-induced changes in SREBP-1 and PPAR- $\alpha$ expression}

SREBP-1 and PPAR- $\alpha$ were evaluated in association with the mGluR5 blockade to better clarify the changes in fat accumulation. SREBPs are involved in the modulation of cholesterol biosynthesis/uptake and fatty acid biosynthesis; ${ }^{20}$ SREBP-1 mRNA has been
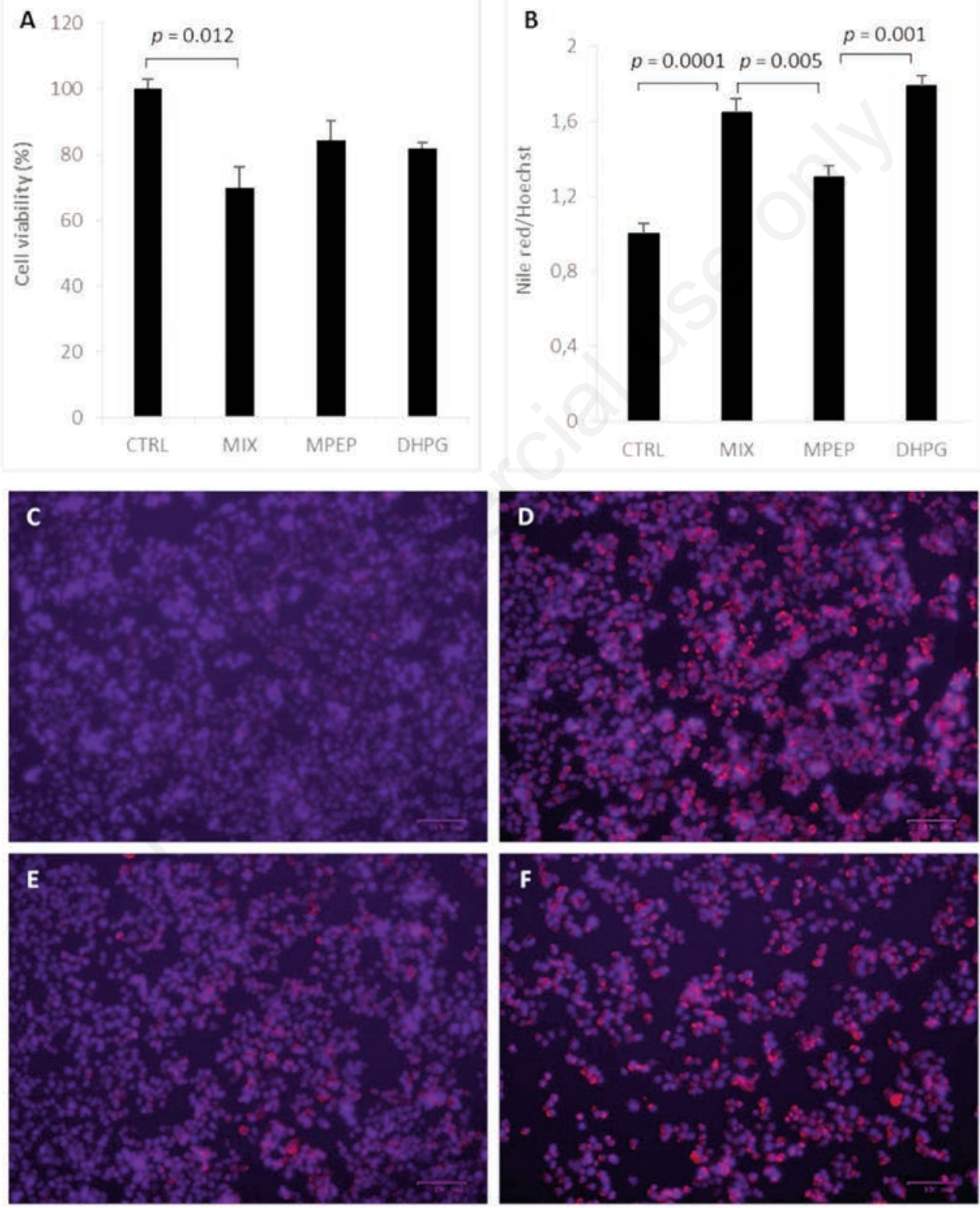

Figure 2. Effect of mGluR5 activation (DHPG) and blockade (MPEP) on cell viability and O/P mix-induced fat accumulation in HepG2 cells. A) $1.5 \mathrm{mM} \mathrm{O/P}$ mixture induced a significant decrease in cell viability in the MIX group in comparison with the CTRL group. B) FA content in HepG2 cells increased significantly when cells were incubated in presence of $1.5 \mathrm{mM} \mathrm{O} / \mathrm{P}$ mixture; the blockade of mGluR5 receptor in O/P treated HepG2 cells prevented FA accumulation. C-F) Hoechst and Nile red uptake in HepG2 cells from CTRL, MIX, MPEP and DHPG groups, respectively; fluorescent probes uptake was evaluated using Image J. The error bars represent the standard error of measurements from three independent experiments each run in duplicate. 
found to be upregulated in both in vitro ${ }^{21,22}$ and in vivo ${ }^{23}$ models of hepatic steatosis. PPAR- $\alpha$ is also considered a major regulator of lipid metabolism in the liver; ${ }^{24} \mathrm{OA}$-induced fat accumulation resulted in a reduced PPAR- $\alpha$ expression in HepG2. ${ }^{25}$ In our model, mGluR5 blockade was associated with a reversion in SREBP-1 and PPAR- $\alpha$ expression changes observed in HepG2 treated with $1.5 \mathrm{mM} \mathrm{O} / \mathrm{P}$ mixture. As expected, the incubation with $\mathrm{O} / \mathrm{P}$ mixture resulted in a significant increase in SREBP-1 and decrease in PPAR- $\alpha$ expression; on the contrary, the mGluR5 blockade restored SREBP-1 and PPAR $\alpha$ to levels similar to those observed in CTRL group. For both SREBP-1 and PPAR- $\alpha$, the DHPG group was not significantly different from the MIX group, although it was significantly different from the MPEP group (Figure 3 A,B).

\section{2-h incubation with $\mathrm{O} / \mathrm{P}$ mixture does not affect iNOS} and caspase- 3 expression or NFkB activation

Hepatocytes, the most represented population of liver cells, are among the most important sources of iNOS. iNOS is almost undetectable in non-pathological conditions and its expression is usually induced in response to stress; it has also been found that hepatocyte-derived iNOS has protective effects on NASH involving the iNOS-mediated modulation of hemeoxygenase (HO)-1 expression. ${ }^{26}$ In our model of hepatocyte fat accumulation, the expression of iNOS was unaltered when comparing MIX and CTRL groups; moreover, the activation/blockade of mGluR5 did not affect iNOS expression levels (Figure 4A). To rule out the activation of inflammatory processes in the MIX group, we also evaluated NFkB activation; as a result, we found that in the MIX group
NFkB activation was not significantly different with respect to the CTRL group. However, the mGluR5 blockade was associated with a significant reduction of NFkB activation (Figure 4B). Caspase-3 activation is considered a prominent event in different experimental models of NAFLD; moreover, the pan-caspase inhibitor VX166 reduced the development of fibrosis in murine NASH models. ${ }^{27}$ We found no significant changes in caspase-3 expression after HepG2 incubation with $\mathrm{O} / \mathrm{P}$ mixture, with or without mGluR5 modulation (Figure 4C). Finally, using $\mathrm{H}_{2}$ DCF-DA to evaluate oxidative stress, we observed that the administration of oleate and palmitate induced the production of reactive oxygen species (ROS), while MPEP treatment lowered ROS levels (Figure 5; Supplementary Figure 2). Considered together, these results suggest that, in our FFA accumulation model, inflammatory processes are not activated and the reduction in FFA accumulation may be related to a modulation of SREBP-1 and PPAR- $\alpha$.

\section{Discussion}

Considering NAFLD rising prevalence at a global level, it is essential to find effective molecular targets suitable as therapeutic options for its treatment. Life style changes are currently among the recommended non-pharmacological therapies for treating NAFLD, even though their long-term sustainability is unsatisfactory due to poor patient compliance. ${ }^{28}$ The recommended pharmacological treatment for patients with NAFLD currently involves the administration of glitazones, bile acids or bile acid analogues, fibrates, vitamin E, metformin, and betaine. However, no drug has
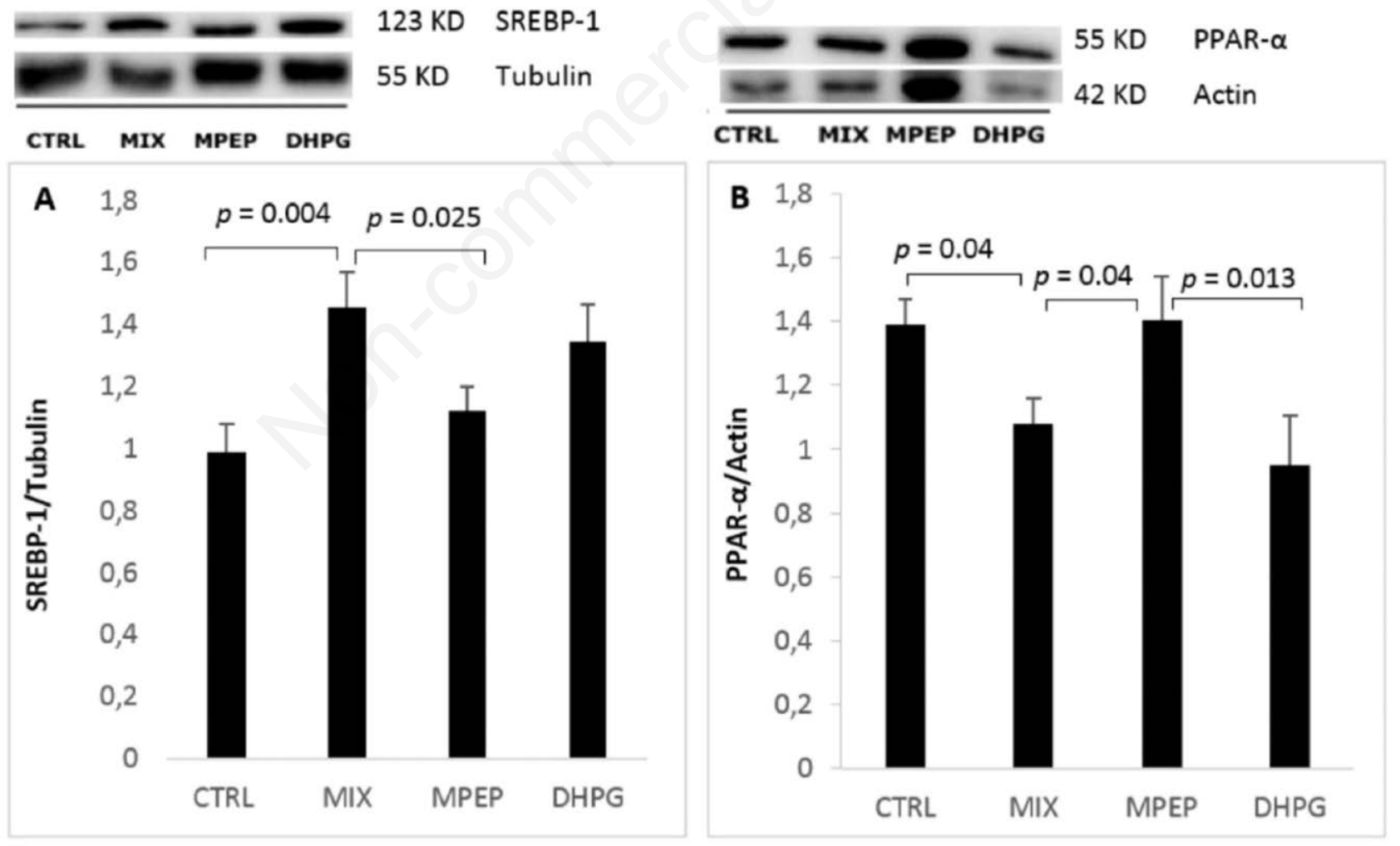

Figure 3. mGluR5 blockade reverts SREBP-1 and PPAR- $\alpha$ expression in HepG2 treated with $1.5 \mathrm{mM}$ O/P mixture. A) The incubation of HepG2 cells with $1.5 \mathrm{mM} \mathrm{O/P}$ mixture induced a significant increase in SREBP-1 protein expression; the blockade of mGluR5 (MPEP group) reduced SREBP-1 to levels similar to the CTRL group; the DHPG group was not significantly different from the MIX group. B) In the MIX group, PPAR- $\alpha$ expression was significantly lower when compared to CTRL; the addition of MPEP to DHPG $100 \mu \mathrm{M}$ prevented the change in MPEP group. The error bars represent the standard error of measurements from three independent experiments each run in duplicate. 
currently passed phase III trials and was approved for NASH by regulatory agencies. ${ }^{29,30}$ FFAs have a pivotal role in the pathogenesis of NAFLD and their hepatic levels are closely related to NAFLD severity. ${ }^{31}$ In this work, we showed for the first time that MPEP, a negative mGluR5 modulator, reduces FFA accumulation in HepG2 human hepatoma cells incubated with a $1.5 \mathrm{mM} \mathrm{O} / \mathrm{P}$ mixture. We also found that MPEP administration is associated with a reversion of O/P-induced changes in SREBP-1 and PPAR$\alpha$ expression and that these changes are independent from inflam- matory processes. The primary goal of this study was to investigate the role of mGluR5 in FFA accumulation in HepG2 human hepatoma cells. The ability of certain substances to reduce FFA accumulation has been previously investigated, especially in models involving a significant degree of cytotoxicity. In a study on HepG2 cells, 24-h treatment with a 2:1 OP mixture was associated with FFA accumulation as well as the upregulation of inflammatory cytokines, such as TNF- $\alpha$, IL- 6 and IL- $1 \beta$; a reduced activation of NFkB was also observed. In the same work, gardenoside treat-

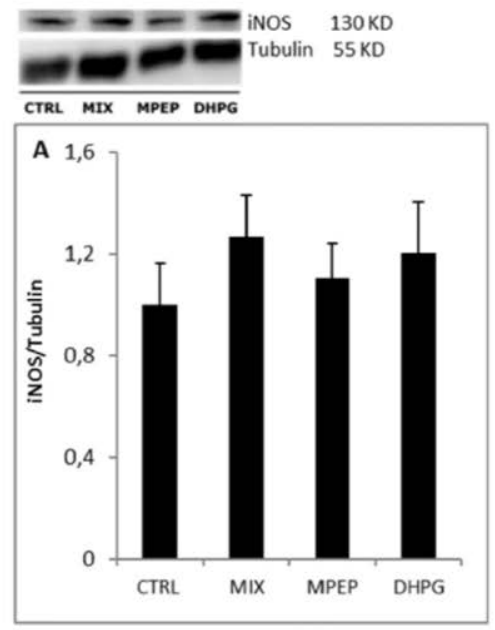

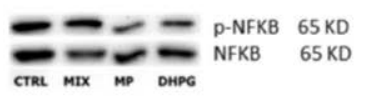

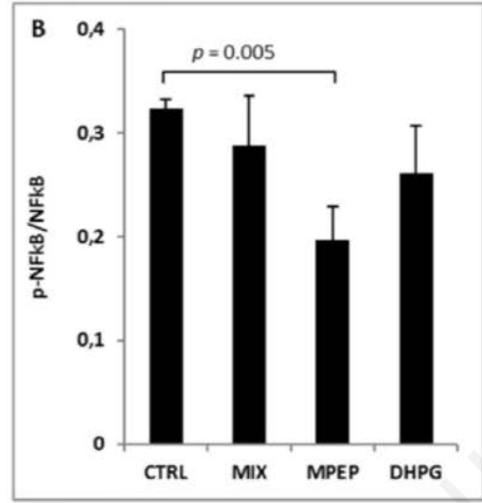

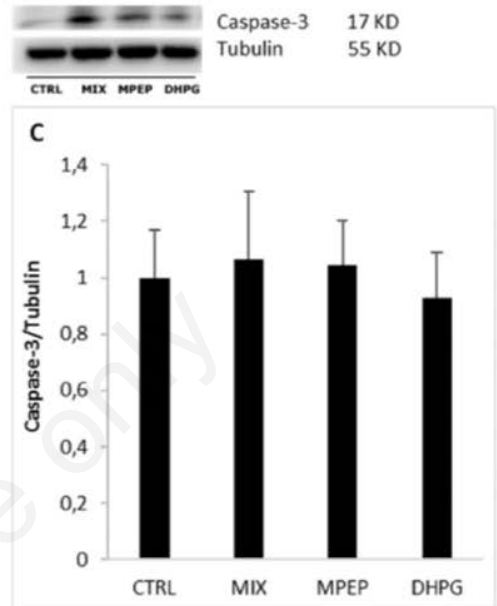

Figure 4. iNOS, NFkB and cleaved caspase-3 expression/activation does not change in HepG2 cells incubated with $1.5 \mathrm{mM}$ O/P mixture. A) HepG2 incubation with $1.5 \mathrm{mM} \mathrm{O/P} \mathrm{mixture} \mathrm{or} \mathrm{mGluR5} \mathrm{agonist/negative} \mathrm{modulator} \mathrm{did} \mathrm{not} \mathrm{induce} \mathrm{significant} \mathrm{changes} \mathrm{in}$ iNOS expression levels. B) HepG2 incubation with $1.5 \mathrm{mM} \mathrm{O} / \mathrm{P}$ mixture did not alter NFkB activation levels; mGluR5 blockade induced a significant reduction in NF-kB activation. C) No changes where observed in cleaved caspase-3 expression. The error bars represent the standard error of measurements from three independent experiments each run in duplicate.
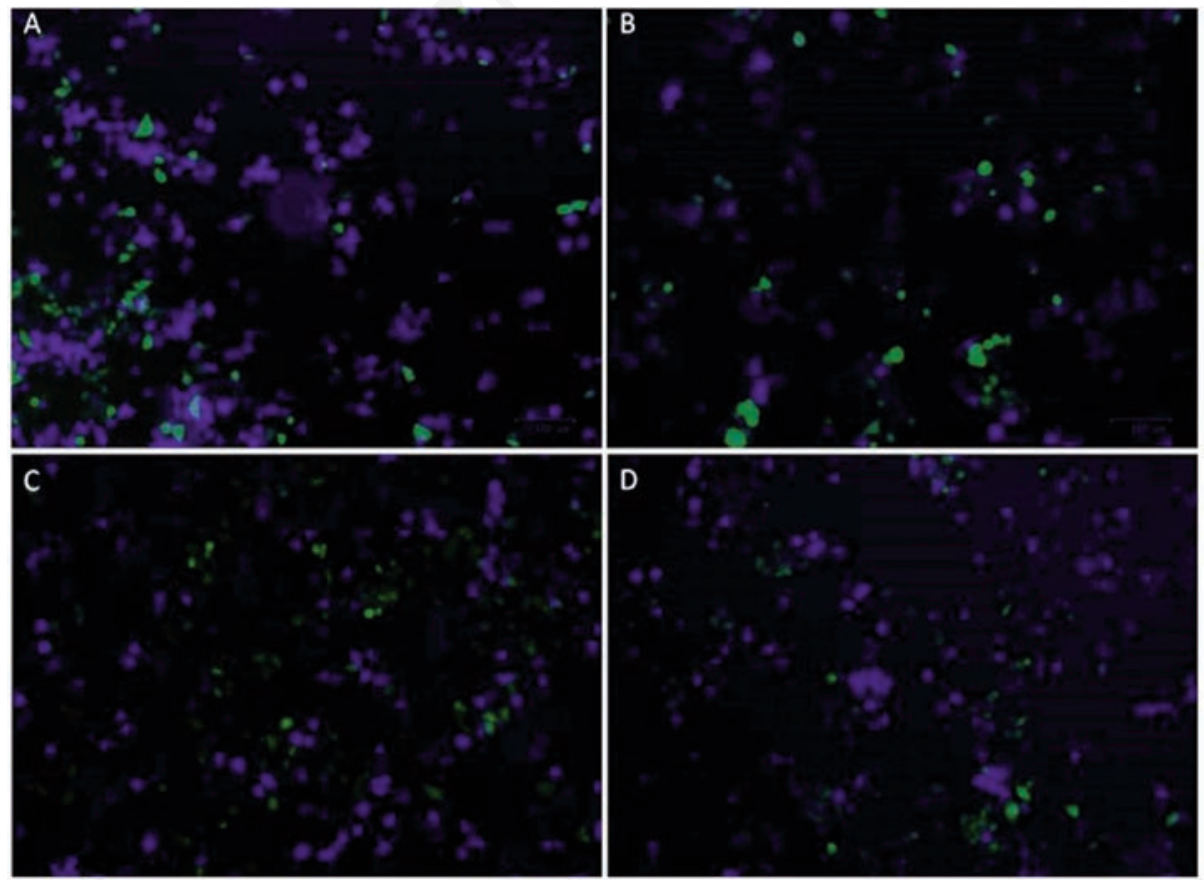

Figure 5. Effect of mGluR5 activation (DHPG) and blockade (MPEP) on O/P mix-induced ROS production in HepG2 cells. Hoechst and $\mathrm{H}_{2}$ DCF-DA uptake in HepG2 cells from CTRL, MIX, MPEP and DHPG groups, respectively. Fluorescent probes uptake was evaluated using Image $\mathrm{J}$. 
ment resulted in a 2-fold decrease in FFAs possibly related, according to the authors, to the anti-inflammatory effect of gardenoside itself. ${ }^{32}$ In a similar work, glycyrrhetinic acid, a licorice extract credited of anti-inflammatory properties in hepatitis, was found to reduce FFA accumulation in HepG2 cells. Also in this work, the FFA-reducing effect was related by the authors to glycyrrhetinic acid ability to stabilize lysosomal membranes, to inhibit cathepsin B and mitochondrial cytochrome c release and to reduce FFA-induced oxidative stress. ${ }^{31}$ In other studies, a higher proportion of palmitic acid is used to induce the inflammatory injury. ${ }^{33}$ In our study, we selected a 12-h, 2:1 OP treatment; the reduced incubation time, associated with the low proportion of palmitic acid resulted in minor/absent lipotoxic effects, thus representing a cellular model of FFA accumulation mimicking benign asymptomatic chronic steatosis. ${ }^{33}$ The incubation of HepG2 cells with 2:1 OP resulted in a 1.6-fold increase in FFA accumulation; however, iNOS and cleaved caspase-3 expression, and NFkB activation state did not change in comparison with control cells, suggesting that in our model inflammatory processes are not yet activated. The pharmacological blockade of mGluR5 by MPEP administration resulted in a significant reduction in FFA accumulation, so that MPEP-group FFA content was comparable to the control cells. Glutamate role at the central level in the metabolic homeostasis has long since been established; in a study, the injection of monosodium glutamate into newborn mice resulted in central obesity and moderate to severe NAFLD at 6 -month age. ${ }^{34}$ The current explanation is that high doses of monosodium glutamate have neurotoxic effects in the hypothalamic arcuate nucleus (ARC). ARC neurons are crucial for the regulation of metabolic homeostasis, including insulin secretion and action. ${ }^{35}$ It has also been shown that mGluR5 modulates central reward pathways and modulates feeding stimulus: selective mGluR5 negative modulation reduced food intake in mice after food deprivation and in obese rats, ${ }^{36}$ selective mGluR5 negative modulation also reduced high-palatable food intake in baboons..$^{37}$ Conversely, intracerebroventricularly injected CHPG, an mGluR5 agonist, induced a dose-dependent stimulation of food intake in rodents. ${ }^{38}$ These studies prompted a Phase I clinical trial to test GET73, a selective mGluR5 negative modulator, as a novel therapeutic agent to treat neuropsychiatric disorders, including alcohol abuse and obesity. ${ }^{39}$ If many studies have been published on the role of mGluR5 in central modulation of appetite and obesity, the same cannot be said on the investigation of mGluR5 role in peripheral organs such as the liver. At the best of our knowledge, only one paper investigated the role of mGluR5 in alcoholic steatosis; in this work, Choi and colleagues demonstrated the alcohol-induced selective expression of mGluR5 in hepatic stellate cells (HSCs), stimulating 2-arachidonoylglycerol (2-AG) production, a molecule promoting de novo lipogenesis in alcoholic liver disease. ${ }^{17}$ We have previously shown that mGluR5 is expressed in primary mice and rat hepatocytes and in human HepG2 cells ${ }^{7,12,13}$ Moreover, in this work, we have shown that FFA accumulation is inhibited by mGluR5 selective blockade in a HepG2-based model of non-inflammatory fat accumulation, showing that lipid metabolism and uptake may be regulated by this receptor without the intervention of HSCs.

SREBP-1 is a transcriptional factor known to regulate the expression of hepatic lipogenic enzymes reported to play an important role in the pathogenesis of NAFLD. ${ }^{40}$ SREBP-1 upregulation has been associated to oleate or palmitate administration in in vitro NAFLD/NASH models ${ }^{21,22,41}$ and to fructose or leptin administration in in vivo NAFLD models. ${ }^{23,42}$ On the other hand, mice with genetic deletion of PPAR- $\alpha$ are more prone to hepatic triglycerides hyperaccumulation when fed with a high fat diet, developing a significantly higher NAFLD activity score compared to WT controls. ${ }^{43-45}$ PPAR- $\alpha$ knockout mice fed with a high fat diet also have increased markers of oxidative stress, inflammation, and cell death. ${ }^{46}$ A reduced PPAR- $\alpha$ expression was also observed in an oleic acid-induced steatosis in HepG2. ${ }^{25}$ In this work, MPEP administration was associated to SREBP-1 downregulation and PPAR- $\alpha$ upregulation in OP-treated HepG2, restoring in both cases values not dissimilar to those found in CTRL group, in concomitance with a reduced FFA accumulation. It has been shown in NAFLD that mTOR, controlling the nuclear localization of both lipin1 and nuclear receptor corepressor 1 (NCoR1), promotes SREBP-1 upregulation and PPAR- $\alpha$ downregulation, respectively. ${ }^{47-49}$ To date, no work has been published demonstrating that the mTOR pathway is a downstream target of mGluR5 in the liver. However, mGluR5 has been found to activate the mTOR pathway in hippocampal slices, a mechanism that seems to be compromised in Fragile X syndrome, ${ }^{50,51}$ a condition associated with a loss of visceral adiposity. ${ }^{52}$ It is possible then that the same pathway might be replicated also in hepatocytes, thus justifying the effects on SREBP-1 and PPAR- $\alpha$ expression we observed in MPEP-treated fatty hepatocytes (Figure 6).

It is not possible to extrapolate from our data the exact modalities of mGluR5 activation. A glutamate accumulation may be responsible for the mGluR5 hyperactivation as already seen in the promotion of liver ischemic injury. ${ }^{14,16}$ Recently, serum and liver levels of glutamate/glutamine ratio have been show to increase significantly in patients with NAFLD; the increase in glutamate/glutamine ratio significantly correlated with the severity of NAFLD and fibrosis. ${ }^{53}$ The underlying mechanism for altered glutamate levels and the associated correlation with NAFLD severity is unclear. However, gene expression changes in NAFLD patients suggest that the liver may switch from a relatively low glutamine catabolism by healthy hepatocytes to a significantly higher glutamine consumption in NASH. ${ }^{53}$ These changes may be responsible for a massive conversion of glutamine into glutamate, resulting in an increase in glutamate secretion and mGluR5 hyperactivation. Recently, it has also been shown that a ROS-mediated increase in glutamate secretion occurs in a model of alcohol-induced steatosis, resulting in mGluR5s hyperactivation on stellate cells and abnor-

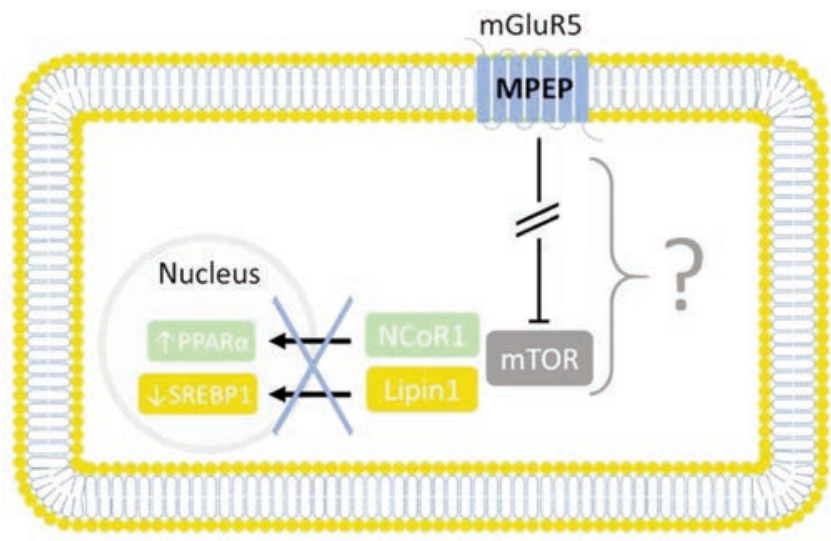

Figure 6. Schematic representation of the hypothetical mechanism proposed for the changes observed in SREBP-1 and PPAR- $\alpha$ protein expression. $m$ TOR has a central role in lipid metabolism; dysregulation of mTOR signaling has been observed in obesity, diabetes, cancer and fatty liver diseases. The modulatory mTOR role involves the modulation of SREBP-1 and PPAR- $\alpha$ expression. mGluR5 has been found to activate the mTOR pathway in hippocampal slices, suggesting that the same pathway might be replicated also in hepatocytes. 
mal fat accumulation in hepatocytes. ${ }^{17}$ In addition to fatty acid accumulation, in our model we also observed an increase in ROS production, which was reversed by the mGluR5 blockade. Surprisingly, also the activation of this receptor significantly reduced the oxidative stress. According to our results, in a mouse model of acetaminophen toxicity, the use of MPEP has been demonstrated to protect against oxidative stress. ${ }^{13}$ However, because of its structure, MPEP may form conjugates with GSH, ${ }^{19}$ minimizing its protective effect. On the other hand, DHPG is able to attenuate glutathione loss in oligodendrocyte precursors, thus attenuating ROS production. ${ }^{54}$

In conclusion, we found for the first time that the pharmacological blockade of mGluR5 reduces FFA accumulation in an in vitro model of steatosis. We also found that the mGluR5 inhibition is associated with a reversion of FFA induced changes in SREBP1 and PPAR- $\alpha$ expression, suggesting that mGluR5 hyperactivation may promote FFA accumulation through changes in the expression of enzymes involved in FFA metabolism and uptake. Glutamate has been found to have increased tissue and serum levels in NAFLD, so explaining mGluR5 hyperactivation. A limitation of the present work is that no precise mechanism can be demonstrated based on the presented data. However, considering that mTOR is the main actor in NAFLD progression and that mGluR5 controls mTOR activation in the CNS, we suggest that a similar mechanism may reasonably occur also in human hepatoma cells.

\section{Acknowledgments}

We thank Ms. Micaela Ascoli for her invaluable administrative managing bureaucratic impediments, and we also thank Ms. Nicoletta Breda for her skilful assistance.

\section{References}

1. LaBrecque DR, Abbas Z, Anania F, Ferenci P, Khan AG, Goh K-L, et al. World Gastroenterology Organisation Global Guidelines. J Clin Gastroenterol 2014;48:467-73.

2. Day CP, James OFW. Steatohepatitis: A tale of two "hits"? Gastroenterology 1998;114:842-5.

3. Boncompagni E, Gini E, Ferrigno A, Milanesi G, Gringeri E, Barni S, et al. Decreased apoptosis in fatty livers submitted to subnormothermic machine-perfusion respect to cold storage. Eur J Histochem 2011;55:e40.

4. Buzzetti E, Pinzani M, Tsochatzis EA. The multiple-hit pathogenesis of non-alcoholic fatty liver disease (NAFLD). Metabolism 2016;65:1038-48.

5. Durand D, Carniglia L, Caruso C, Lasag M. Metabotropic glutamate receptors in peripheral tissues: Implications for toxicology. In: Anxiety disorders. InTech; 2011.

6. Storto M, Capobianco L, Battaglia G, Molinaro G, Gradini R, Riozzi B, et al. Insulin secretion is controlled by mGlu5 metabotropic glutamate receptors. Mol Pharmacol 2006;69:1234-41.

7. Storto M, de Grazia U, Knöpfel T, Canonico PL, Copani A, Richelmi P, et al. Selective blockade of mGlu5 metabotropic glutamate receptors protects rat hepatocytes against hypoxic damage. Hepatology 2000;31:649-55.

8. Nakamura E, Hasumura M, San Gabriel A, Uneyama H, Torii $\mathrm{K}$. New frontiers in gut nutrient sensor research: Luminal Glutamate-sensing cells in rat gastric mucosa. J Pharmacol Sci 2010;112:13-8.
9. Storto M, Sallese M, Salvatore L, Poulet R, Condorelli DF, Dell'Albani P, et al. Expression of metabotropic glutamate receptors in the rat and human testis. J Endocrinol 2001;170:71-8.

10. Gill S, Barker M, Pulido O. Neuroexcitatory targets in the female reproductive system of the nonhuman primate (Macaca fascicularis). Toxicol Pathol 2008;36:478-84.

11. Sureda F, Copani A, Bruno V, Knopfel T, Meltzger G, Nicoletti F. Metabotropic glutamate receptor agonists stimulate polyphosphoinositide hydrolysis in primary cultures of rat hepatocytes. Eur J Pharmacol 1997;338:R1-2.

12. Storto M, Battaglia G, Gradini R, Bruno V, Nicoletti F, Vairetti M. Mouse hepatocytes lacking mGlu5 metabotropic glutamate receptors are less sensitive to hypoxic damage. Eur J Pharmacol 2004;497:25-7.

13. Storto M, Ngomba RT, Battaglia G, Freitas I, Griffini P, Richelmi P, et al. Selective blockade of mGlu5 metabotropic glutamate receptors is protective against acetaminophen hepatotoxicity in mice. J Hepatol 2003;38:179-87.

14. Ferrigno A, Berardo C, Di Pasqua LG, Siciliano V, Richelmi P, Nicoletti F, et al. Selective blockade of the metabotropic glutamate receptor mGluR5 protects mouse livers in in vitro and ex vivo models of ischemia reperfusion injury. Int $\mathrm{J}$ Mol Sci 2018;19:314.

15. Chan TS, Cassim S, Raymond V-A, Gottschalk S, Merlen G, Zwingmann C, et al. Upregulation of Krebs cycle and anaerobic glycolysis activity early after onset of liver ischemia. PLoS One 2018;13:e0199177.

16. Nicoletti F, Battaglia G, Storto M, Ngomba RT, Iacovelli L, Arcella A, et al. Metabotropic glutamate receptors: beyond the regulation of synaptic transmission. Psychoneuroendocrinology 2007;32:S40-5.

17. Choi WM, Kim HH, Kim MH, Cinar R, Yi HS, Eun HS, et al. Glutamate signaling in hepatic stellate cells drives alcoholic steatosis. Cell Metab 2019;30:877-89.e7.

18. Berardo C, Siciliano V, Pasqua LG Di, Richelmi P, Vairetti M, Ferrigno A. Comparison between lipofectamine RNAiMAX and GenMute transfection agents in two cellular models of human hepatoma. Eur J Histochem 2019;63:3048.

19. Ferrigno A, Berardo C, Di Pasqua LG, Siciliano V, Richelmi P, Vairetti M. Localization and role of metabotropic glutamate receptors subtype 5 in the gastrointestinal tract. World J Gastroenterol 2017;23:4500-7.

20. Brown MS, Goldstein JL. The SREBP pathway: Regulation of cholesterol metabolism by proteolysis of a membrane-bound transcription factor. Cell 1997;89:331-40.

21. Lounis MA, Bergeron KF, Burhans MS, Ntambi JM, Mounier C. Oleate activates SREBP-1 signaling activity in SCD1-deficient hepatocytes. Am J Physiol Endocrinol Metab 2017;313:E710-20.

22. Liu JF, Ma Y, Wang Y, Du ZY, Shen JK, Peng HL. Reduction of lipid accumulation in HepG2 cells by luteolin is associated with activation of AMPK and mitigation of oxidative stress. Phyther Res 2011;25:588-96.

23. Zhang C, Chen X, Zhu RM, Zhang Y, Yu T, Wang H, et al. Endoplasmic reticulum stress is involved in hepatic SREBP-1c activation and lipid accumulation in fructose-fed mice. Toxicol Lett $2012 ; 212: 229-40$.

24. Desvergne B, IJpenberg A, Devchand PR, Wahli W. The peroxisome proliferator-activated receptors at the cross-road of diet and hormonal signalling. J Steroid Biochem Mol Biol 1998;65:65-74.

25. Cui W, Chen SL, Hu KQ. Quantification and mechanisms of oleic acid-induced steatosis in HepG2 cells. Am J Transl Res 2010;2:95-104. 
26. Qiao Y, Li X, Zhang X, Xiao F, Zhu Y, Fang Z, et al. Hepatocellular iNOS protects liver from NASH through Nrf2dependent activation of HO-1. Biochem Biophys Res Commun 2019;514:372-8.

27. Kanda T, Matsuoka S, Yamazaki M, Shibata T, Nirei K, Takahashi H, et al. Apoptosis and non-alcoholic fatty liver diseases. World J Gastroenterol 2018;24:2661-72.

28. Catalano D, Trovato GM, Martines GF, Randazzo M, Tonzuso A. Bright liver, body composition and insulin resistance changes with nutritional intervention: A follow-up study. Liver Int 2008;28:1280-7.

29. European Association for the Study of the Liver (EASL), European Association for the Study of Diabetes (EASD), European Association for the Study of Obesity (EASO). EASL-EASD-EASO Clinical Practice Guidelines for the management of non-alcoholic fatty liver disease. J Hepatol 2016;64:1388-402.

30. Francque S, Vonghia L. Pharmacological treatment for nonalcoholic fatty liver disease. Adv Ther 2019;36:1052-74.

31. Wu X, Zhang L, Gurley E, Studer E, Shang J, Wang T, et al. Prevention of free fatty acid-induced hepatic lipotoxicity by $18 \beta$-glycyrrhetinic acid through lysosomal and mitochondrial pathways. Hepatology 2008;47:1905-15.

32. Liang $\mathrm{H}$, Zhang L, Wang $\mathrm{H}$, Tang J, Yang J, Wu C, et al. Inhibitory effect of gardenoside on free fatty acid-induced steatosis in HepG2 hepatocytes. Int J Mol Sci 2015;16:27749-56.

33. Gómez-Lechón MJ, Donato MT, Martínez-Romero A, Jiménez N, Castell JV, O'Connor JE. A human hepatocellular in vitro model to investigate steatosis. Chem Biol Interact 2007;165:106-16.

34. Nakanishi Y, Tsuneyama K, Fujimoto M, Salunga TL, Nomoto $\mathrm{K}$, An JL, et al. Monosodium glutamate (MSG): A villain and promoter of liver inflammation and dysplasia. J Autoimmun 2008;30:42-50.

35. Hernández Bautista RJ, Mahmoud AM, Königsberg M, López Díaz Guerrero NE. Obesity: Pathophysiology, monosodium glutamate-induced model and anti-obesity medicinal plants. Biomed Pharmacother 2019;111:503-16.

36. Bradbury MJ, Campbell U, Giracello D, Chapman D, King C, Tehrani L, et al. Metabotropic glutamate receptor mGlu5 is a mediator of appetite and energy balance in rats and mice. J Pharmacol Exp Ther 2005;313:395-402.

37. Bisaga A, Danysz W, Foltin RW. Antagonism of glutamatergic NMDA and mGluR5 receptors decreases consumption of food in baboon model of binge-eating disorder. Eur Neuropsychopharmacol 2008;18:794-802.

38. Ploj K, Albery-Larsdotter S, Arlbrandt S, Kjaer MB, Skantze PMC, Storlien LH. The metabotropic glutamate mGluR5 receptor agonist CHPG stimulates food intake. Neuroreport 2010;21:704-8.

39. Haass-Koffler CL, Goodyear K, Long VM, Tran HH, Loche A, Cacciaglia R, et al. A Phase I randomized clinical trial testing the safety, tolerability and preliminary pharmacokinetics of the mGluR5 negative allosteric modulator GET 73 following single and repeated doses in healthy volunteers. Eur J Pharm Sci 2017;109:78-85.
40. Ahmed MH, Byrne CD. Modulation of sterol regulatory element binding proteins (SREBPs) as potential treatments for non-alcoholic fatty liver disease (NAFLD). Drug Discov Today 2007;12:740-7.

41. Dhami-Shah H, Vaidya R, Udipi S, Raghavan S, Abhijit S, Mohan V, et al. Picroside II attenuates fatty acid accumulation in HEPG2 cells via modulation of fatty acid uptake and synthesis. Clin Mol Hepatol 2018;24:77-87.

42. Wu L, Chen G, Liu W, Yang X, Gao J, Huang L, et al. Intramuscular injection of exogenous leptin induces adiposity, glucose intolerance and fatty liver by repressing the JAK2STAT3/PI3K pathway in a rat model. Gen Comp Endocrinol 2017;252:88-96.

43. Abdelmegeed MA, Yoo S-H, Henderson LE, Gonzalez FJ, Woodcroft KJ, Song B-J. PPAR $\alpha$ expression protects male mice from high fat-induced nonalcoholic fatty liver. J Nutr 2011;141:603-10.

44. Stienstra R, Mandard S, Patsouris D, Maass C, Kersten S, Müller M. Peroxisome proliferator-activated receptor $\alpha$ protects against obesity-induced hepatic inflammation. Endocrinology 2007;148:2753-63.

45. Patsouris D, Reddy JK, Müller M, Kersten S. Peroxisome proliferator-activated receptor $\alpha$ mediates the effects of high-fat diet on hepatic gene expression. Endocrinology 2006; 147:1508-16.

46. Stienstra R, Duval C, Müller M, Kersten S. PPARs, obesity, and inflammation. PPAR Res 2007;2007:95974.

47. Soliman GA. The integral role of $\mathrm{mTOR}$ in lipid metabolism. Cell Cycle 2011;10:861-2.

48. Lamming DW, Sabatini DM. A central role for mTOR in lipid homeostasis. Cell Metab 2013;18:465-9.

49. Kim K, Pyo S, Um SH. S6 kinase 2 deficiency enhances ketone body production and increases peroxisome proliferatoractivated receptor alpha activity in the liver. Hepatology 2012;55:1727-37.

50. Huber KM, Klann E, Costa-Mattioli M, Zukin RS. Dysregulation of mammalian target of rapamycin signaling in mouse models of autism. J Neurosci 2015;35:13836-42.

51. Ronesi JA, Collins KA, Hays SA, Tsai NP, Guo W, Birnbaum SG, et al. Disrupted Homer scaffolds mediate abnormal mGluR5 function in a mouse model of fragile X syndrome. Nat Neurosci 2012;15:431-40.

52. Leboucher A, Bermudez-Martin P, Mouska X, Amri EZ, Pisani DF, Davidovic L. Fmr1-deficiency impacts body composition, skeleton, and bone microstructure in a mouse model of Fragile X syndrome. Front Endocrinol (Lausanne) 2019;10:678.

53. Du K, Chitneni SK, Suzuki A, Wang Y, Henao R, Hyun J, et al. Increased glutaminolysis marks active scarring in nonalcoholic steatohepatitis progression. Cell Mol Gastroenterol Hepatol 2020;10:1-21.

54. Deng W, Wang H, Rosenberg PA, Volpe JJ, Jensen FE. Role of metabotropic glutamate receptors in oligodendrocyte excitotoxicity and oxidative stress. PNAS 2004;101:7751-6.

Received for publication: 25 August 2020. Accepted for publication: 2 October 2020.

This work is licensed under a Creative Commons Attribution-NonCommercial 4.0 International License (CC BY-NC 4.0).

(C) Copyright: the Author(s), 2020

Licensee PAGEPress, Italy

European Journal of Histochemistry 2020; 64:3175

doi:10.4081/ejh.2020.3175 